\title{
Evidence-based medicine in urology
}

\author{
Philipp Dahm ${ }^{1,2} \cdot$ Frank Kunath $^{3,4}$
}

Published online: 28 February 2020

(c) This is a U.S. Government work and not under copyright protection in the US; foreign copyright protection may apply 2020

Keywords Evidence-based medicine · Urology · GRADE · Systematic reviews · Guidelines

Evidence-based medicine (EBM) is a term coined by Gordon Guyatt at McMaster University in 1991 referring to the use of the current best evidence from clinical research to the care of individual patients [1]. Its first guiding principle refers to a hierarchy of evidence; meaning that some evidence is more trustworthy than others [2]. The second, equally important principle of EBM relates to the need to integrate the current best evidence with an individual patient's circumstances, values, and preferences. There is, therefore, no automatism from evidence, even if of high quality, to clinical action. A pragmatic and transparent approach, both rating the certainty of evidence and to moving from evidence to decisions has since been provided by the GRADE Working Group [3, 4]. The role of the empathetic expert clinician and urologic surgeon is, therefore, not under threat by EBM; it is his/her role to help the patient find the 'best' management approach [5].

Urology has broadly embraced EBM as particularly witnessed in the sphere of clinical practice guidelines with professional organizations such as the American Urological Association (AUA), the European Urological Association (EAU), and the German Society of Urology with its UroEvidence Group making major investments towards the development of evidence-based guidance document based on high-quality systematic reviews [6-8]. EBM has also

Philipp Dahm

pdahm@umn.edu

1 Urology Section, Minneapolis Veterans Healthcare Healthcare System, 112D One Veterans Drive, Minneapolis, MN 55417, USA

2 Department of Urology, University of Minnesota, Minneapolis, USA

3 Department of Urology and Pediatric Urology, Friedrich-A lexander-Universität Erlangen-Nürnberg (FAU), University Hospital Erlangen, Krankenhausstrasse 12, 91054 Erlangen, Germany

4 UroEvidence@Deutsche Gesellschaft für Urologie, Berlin, Germany made a firm entry into urology residency education as witnessed by the AUA's core curriculum and the widespread availability of urology-specific resources such as Users' Guide to the Urology Literature [9]. Clearly, EBM is here in urology to stay.

In this special edition of the "World Journal of Urology", we are privileged to provide a dedicated forum for a group of recognized clinical experts to review the current best evidence on a broad spectrum of hot topics in prostate cancer. The selected themes span the horizon from the translational research to comparative effectiveness; from early stage, clinically localized prostate cancer to terminal, castraterefractory disease with representation of the central EBM themes of prevention, therapy, diagnosis, and prognosis [2]. Each of the author teams has critically appraised the current best evidence on their topic and summarized those aspects for us that are the most clinically relevant.

In the first two articles, Ivo Shoots and colleagues review the role of multivariate risk prediction models without and with formal integration of MRI in making the diagnosis of clinically significant prostate cancer; a timely topic that has only recently been the subject of several important studies in this journal $[10,11]$. The submission by Narayan adds to this a critical assessment of individual biomarkers that have recently become available to arrive at 'smarter' screening for prostate cancer [12]. For the treatment of clinically localized prostate cancer, Luke Lavaleé reviews the best practice protocols for active surveillance, Tiffany Daly describes the evolution of radiotherapy options, and Philippe Violette and colleagues summarize evidence-based, risk-adapted guidance for perioperative venous thrombosis prophylaxis. On the topic of advanced prostate cancer, Kunath and colleagues and Pinart and colleagues report systematic reviews for early versus deferred androgen deprivation therapy and prediction models for castrate-refractory prostate cancer, respectively. Finally, in the last two articles, Thomas Worst and colleagues and Sven Wach and colleagues review 
groundbreaking research as it relates to prostate cancer gene expression and androgen reception splice variants.

We are hopeful that these articles will inspire a thoughtful and engaged discussion about the future direction of prostate cancer diagnosis and management, promote the cause of EBM in urology and ultimately translate into higher quality, evidence-based care for our patients.

\section{References}

1. Sur RL, Dahm P (2011) History of evidence-based medicine. Indian J Urol 27:487-489

2. Dahm P, Scales CD, Sur RL et al (2006) An introduction to evidence-based medicine in urology. AUA Update Ser 25:230-235

3. Alonso-Coello P, Oxman AD, Moberg J et al (2016) GRADE Evidence to Decision (EtD) frameworks: a systematic and transparent approach to making well informed healthcare choices. Clinical practice guidelines. BMJ 353:i2089

4. Alonso-Coello P, Schunemann HJ, Moberg J et al (2016) GRADE Evidence to Decision (EtD) frameworks: a systematic and transparent approach to making well informed healthcare choices. 1: Introduction. BMJ 353:i2016

5. Canfield SE, Dahm P (2010) Evidence-based urology in practice: incorporating patient values in evidence-based clinical decision making. BJU Int 105:4-5

6. Faraday M, Hubbard H, Kosiak B et al (2009) Staying at the cutting edge: a review and analysis of evidence reporting and grading; the recommendations of the American Urological Association. BJU Int 104:294-297

7. Knoll T, Omar MI, Maclennan S et al (2018) Key steps in conducting systematic reviews for underpinning clinical practice guidelines: methodology of the European Association of Urology. Eur Urol 73:290-300

8. Kranz J, Kunath F, Borgmann H et al (2014) "UroEvidence" Centre for knowledge translation of the DGU (German Society of Urology). Summarizing, analysing and making current knowledge available. Urol A 53:83-86

9. Dahm P, Preminger GM (2007) A users guide to the urological literature: introducing a series of evidence based medicine review articles. J Urol 178:1149

10. Tokas T, Junker D, Nagele U (2019) One-stop biparametric MRI and MRI/ultrasound fusion-guided biopsy: can we include the histopathology result and our treatment recommendations in our diagnostic pathway? World J Urol. https://doi.org/10.1007/s0034 5-019-02940-2

11. Wetterauer C, Winkel DJ, Federer-Gsponer JR et al (2019) Novices in MRI-targeted prostate biopsy benefit from structured reporting of MRI findings. World J Urol. https://doi.org/10.1007/ s00345-019-02953-x

12. Tan GH, Nason G, Ajib K et al (2019) Smarter screening for prostate cancer. World J Urol 37:991-999

Publisher's Note Springer Nature remains neutral with regard to jurisdictional claims in published maps and institutional affiliations. 